NSM 00940

\title{
Somatosensory evoked potentials of the dog: recording techniques and normal values
}

\author{
Chris W. Vanderzant ${ }^{1}$, Robert J. Schott ${ }^{2}$, JoAnne E. Natale ${ }^{3}$. \\ Carol A. Pondo ${ }^{1}$ and Louis G. D'Alecy ${ }^{2.3}$ \\ Departments of 'Neurology, 'Surgery and "Physiologv, University of Michigan Medical School. Ann Arbor, MI 48lo9.(0)36 (U.S.A.) \\ (Received 20 May 1988) \\ (Accepted 18 October 1988)
}

Key words: Somatosensory evoked potential; Dog; Stimulation technique; Recording technique: Normative data

\begin{abstract}
Median and tibial nerve somatosensory evoked potentials (SSEPs) of 5 sedated dogs were studied to determine their normal features and optimal stimulation and recording techniques. Cortical potentials were mapped from an extensive array of skull electrodes as each limb was independently stimulated with subdermal needles. The effects of bandpass and stimulus intensity and rate were also assessed. Three cortical components (P1, N1, P2) were evoked by median or tibial nerve stimulation and were localized along the coronal suture at lateral and medial electrodes, respectively. SSEP voltage varied much more than morphology, topography. or latency. The inion was a stable, indifferent reference site. Cortical SSEP frequency content was mostly below $250 \mathrm{~Hz}$. Maximal SSEP voltage was achieved only at stimulus intensities 2-3 times motor threshold. Appropriate methods minimize technical difficulties and consistently yield legible SSEPs.
\end{abstract}

\section{Introduction}

Short-latency somatosensory evoked potentials (SSEPs) are a sensitive quantitative measure of conduction in somatosensory pathways of the central nervous system. SSEPs are now widely used in clinical medicine to diagnose abnormal sensory function and to monitor neurologic status. However, the myriad SSEP techniques adopted by investigators have presented a major obstacle to their clinical application. Recently, guidelines were published in an effort to standardize techniques and nomenclature for clinical evoked potential studied of humans (American EEG Society, 1984).

SSEPs are increasingly used in research involving animals, especially to quantify cerebral dys-

Correspondence: L.G. D'Alecy, Medical Science II 7799. University of Michigan Medical School. Ann Arbor. MI 481090622. U.S.A. function in models of brain ischemia. These SSEP studies also show little uniformity of stimulus or recording technique, nomenclature, or methods of data analysis and presentation. Some standardization would be helpful to investigators less experienced in SSEP methods, allow closer comparisons between studies, and perhaps ultimately facilitate a better understanding of the relation of SSEP changes to cerebral pathology (Ropper, 1986). We studied the SSEPs of sedated dogs to determine optimal stimulation and recording techniques and to obtain normative data for future studies employing the SSEP as an adjunctive measure of the cerebral effects of ischemia.

\section{Materials and Methods}

Five healthy and fed adult mongrel dogs weighing $15.4-24.0 \mathrm{~kg}$ ( 4 male, 1 female) were studied. 
Each was anesthetized with sodium pentobarbital $30 \mathrm{mg} / \mathrm{kg}$ intravenously, intubated, and maintained on room air with controlled ventilation. Neuromuscular blocking agents were not used. An esophageal probe was inserted to the level of the heart to monitor body temperature, and heating lamps were used to correct hypothermia. The electrocärdiogram, respirations, and mean and pulsatile arterial blood pressure were continuously monitored.

The anesthetized animal's scalp was incised along the mid-dorsal line and reflected to the squamous (parietotemporal) sutures, widely exposing the skull bilaterally. Recording electrodes consisting of $1 / 4$ inch self-tapping stainless steel screws attached to teflon-insulated cable were inserted into drilled holes without penetration of the dura. An array of at least 17 electrodes was placed in each dog's skull (Fig. 1) using 3 anatomical landmarks for consistent placement: (1) anteriorly, the point on the pars frontalis where the external frontal crests diverge anterolaterally from the sagittal crest; (2) posteriorly, the inion (central surface point on the external occipital protuberance); and (3) the squamous sutures laterally. Five

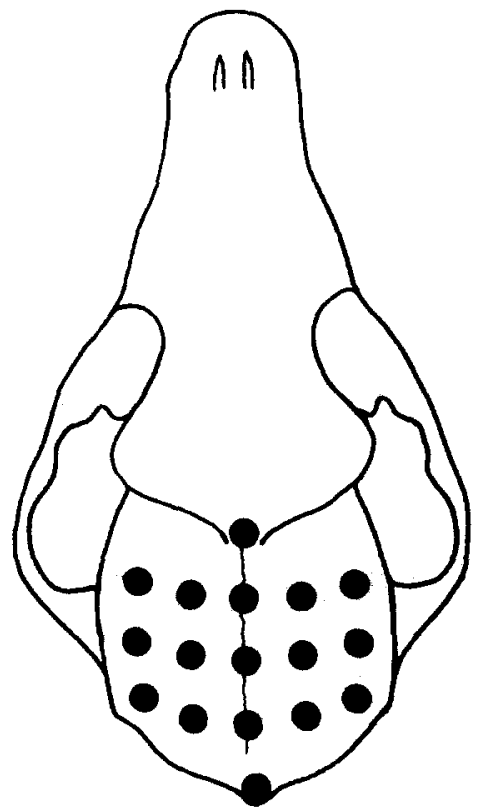

Fig. 1. Dorsal view of dog skull, showing minimum array of skull electrodes for SSEP recordings. electrodes were placed along the mid-dorsal line at equal inter-electrode distances, extending from the divergence of the external frontal crests to the inion. To each side of the 3 intermediate midline electrodes were placed 2 or 3 electrodes at equal intervals, forming 3 parallel rows of electrodes extending transversely between the syuamous sutures. In 3 dogs, 8-16 additional electrodes were placed near the anterior transverse row to map SSEP topography in greater detail. Intial recordings utilized a limb or lumbar spine clectrode as reference, but once the absence of cortical SSEPs at the occiput was established. an inion reference was used because of its greater stability and lesser myogenic activity.

In 2 animals, electrodes were also inserted at cervical, thoracic, and lumbar spinal levels to record the spine potentials evoked by tibial nerve stimulation. These consisted of $5 / 8$ inch stainless steel screws drilled into spinous processes or EEG needle electrodes inserted between them.

Electrode impedances were maintained below $5000 \Omega$. The ground was a needle electrode placed proximally in the stimulated limb. Four-channel recordings were obtained on a Nicolet Compact Four system. Amplifier gain was 24,000, and amplifier bandpass was $30-3000 \mathrm{~Hz}(\cdots 3 \mathrm{~dB})$ with filter roll-off slopes of $12 \mathrm{~dB} /$ octave. Analysis times were $50 \mathrm{~ms}$ for median nerve and $100 \mathrm{~ms}$ for tibial nerve studies. There were 512 data points per channel, giving dwell times of 98 and $195 \mu \mathrm{s}$ for median and tibial nerve studies, respectively. Amplitude resolution of $A / D$ conversion was 8 bits. Each average consisted of 100-300 samples. repeated at least once for each derivation and stimulus condition to assure coherence. The developing average and raw EEG signal were monitored and averaging stopped if artifacts were prominent.

The stimulus was a $200-\mu \mathrm{s}$ monophasic square wave generated by a constant-current stimulator at a rate of $5.1 \mathrm{~Hz}$. The intensity was well above motor threshold (3-8 $\mathrm{mA}$ ), evoking a moderately vigorous muscle twitch. Stimulating electrodes were standard needle EEG type with impedances below $10,000 \Omega$, inserted subdermally close to the nerve with the cathode $2 \mathrm{~cm}$ proximal to the anode. The median nerve was stimulated in the 
distal antebrachium immediately proximal to the first inter-digital space of the forepaw. The tibial nerve was stimulated $2-3 \mathrm{~cm}$ proximal to the tibiotarsal (ankle) joint, along the tendinous insertion of the gastrocnemius muscle. Each limb was stimulated independently until SSEPs were recorded from all scalp electrodes.

The effect of different filter settings was studied in 3 animals. Once the maximal cortical SSEP was localized, the low frequency (high-pass) filter was held constant at $5 \mathrm{~Hz}$ as repeat recordings were obtained at high frequency (low-pass) filter settings of $30,100,250,500,1000,1500,3000$ and $10,000 \mathrm{~Hz}$. The effects of low frequency filter settings of $0.01,1,5,10,30,100,150$ and $300 \mathrm{~Hz}$ were similarly investigated with the high frequency filter set at $3000 \mathrm{~Hz}$. The effect of stimulus intensity on median and tibial nerve SSEPs was investigated in 3 animals. Using the derivation that best defined cortical SSEPs, repeat recordings were obtained at stimulus intensities ranging from below motor threshold to 4 times the threshold value. The effect of stimulus rate was studied in 2 animals. Cortical SSEPs were recorded at median nerve stimulation rates of $5.1,10.1,15.1$ and 17.5 $\mathrm{Hz}$, and tibial nerve stimulation rates of 5.1, 10.1, and $15.1 \mathrm{~Hz}$, with 30 and $50 \mathrm{~ms}$ analysis times, respectively.

\section{Results}

In all 5 dogs, well-defined and reproducible SSEPs were recorded from the skull overlying somatosensory cortex with median or tibial nerve stimulation. The morphology, topography, and latency of each component showed a high degree of consistency among animals. Each waveform was labeled "N" or " $P$ " to denote surface polarity and numbered sequentially.

SSEP morphology and polarity were identical with median or tibial nerve stimulation (Fig. 2). Three potentials were consistently identified: an initial positive component (P1), a negative component (N1), and then a smaller positivity (P2) that was longer in duration than preceding components. P2 was occasionally followed by low volt-

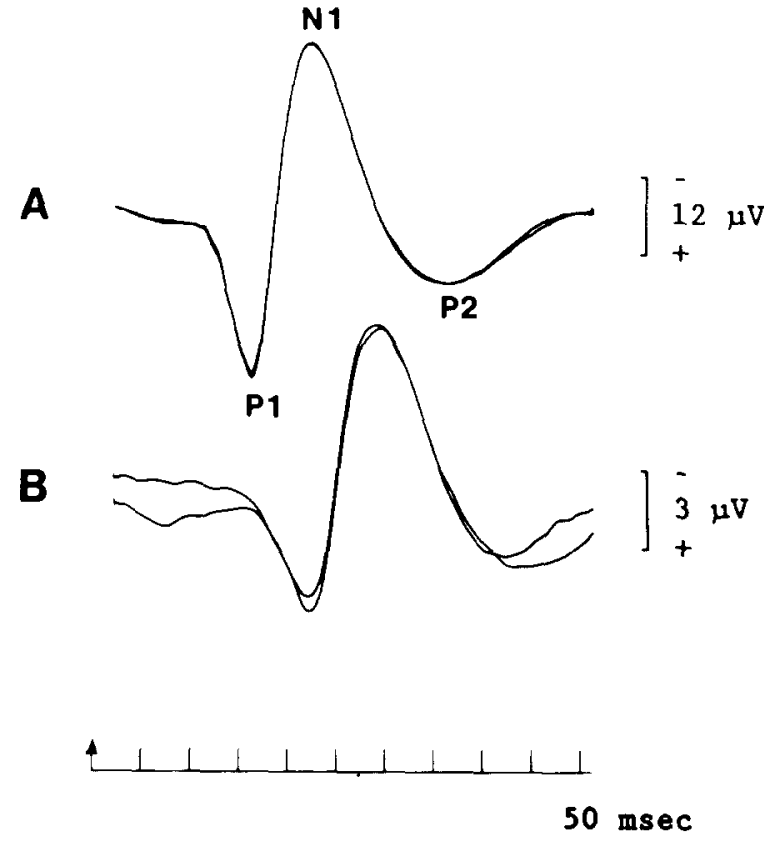

Fig. 2. Typical canine SSEPs recorded at the skull overlying somatosensory cortex on contralateral median (A) and tibial (B) nerve stimulation. In this and all subsequent figures, 2 separate averages of 300 individual responses are superimposed to demonstrate reproducibility. Waveforms are unretouched and no digital filtering was used.

age negative (N2) and positive (P3) components, which were attenuated by sedation.

The voltages of $\mathrm{P} 1, \mathrm{~N} 1$, and $\mathrm{P} 2$ varied much more than peak latencies (Table I). Tibial nerve SSEP latencies were longer, proportional to the greater length of that conduction pathway. Latency variations among animals were small and closely related to differences in body size. But voltages varied greatly among dogs, and sometimes even between left- and right-sided stimulation of the same animal (e.g., dog no. 3). P1, N1, and P2 were 2-4 times greater in amplitude on median nerve stimulation. N1 was the largest component, ranging from 8.2 to $51.1 \mu \mathrm{V}$ when evoked from the median nerve and 3.1 to $34.8 \mu \mathrm{V}$ in tibial studies. P1 showed a similarly wide voltage range. P2 was lowest and least variable in amplitude.

SSEP topography was similar among animals (Fig. 3A, B). On median nerve stimulation, the P1-N1-P2 complex was localized to the contralateral parietal bone, on the dorsolateral aspect 
TABLE I

\section{PEAK LATENCIES AND VOLTAGES OF CORTICAL SOMATOSENSORY EVOKED POTENTIALS}

Latencies in ms and voltages in $\mu \mathrm{V}$. Values from left- and right-sided stimulation are separated by a slash ( $/$ ). Two superimposed, coherent averages from the skull location showing maximal SSEP voltage were measured with cursors. All voltages are from a baseline determined as a function of the entire waveform. S.D. = standard deviation.

\begin{tabular}{|c|c|c|c|c|c|c|c|}
\hline \multicolumn{2}{|l|}{$\overline{D o g}$} & \multicolumn{2}{|l|}{ P1 } & \multicolumn{2}{|l|}{ N1 } & \multicolumn{2}{|l|}{$\mathrm{P} 2$} \\
\hline No. & Wt. (kg) & $\overline{\text { Latency }}$ & Voltage & Latency & Voltage & Latency & Voltage \\
\hline \multicolumn{8}{|c|}{ Median nerve stimulation } \\
\hline 1 & 18.1 & $-\quad / 16.7$ & $-\quad 24.6$ & $\ldots \quad / 23.0$ & $-\quad / 28.6$ & -36.3 & 6.5 \\
\hline 2 & 20.4 & $18.8 / 14.9$ & $10.2 / 6.4$ & $25.3 / 20.0$ & $22.4 / 13.0$ & $37.7 / 32.5$ & $13.0 / 9.3$ \\
\hline 3 & 24.0 & $18.9 / 15.8$ & $49.4 / 10.5$ & $24.8 / 20.5$ & $51.1 / 23.3$ & $38.1 / 32.4$ & $15.4 / 12.2$ \\
\hline 4 & 15.4 & $13.9 / 13.5$ & $5.9 / 5.6$ & $19.1 / 18.4$ & $8.2 / 10.0$ & $30.0 / 29.6$ & $3.0 / 3.9$ \\
\hline 5 & 20.6 & $16.0 / 15.7$ & $28.4 / 23.0$ & $21.8 / 21.6$ & $33.3 / 27.4$ & $34.4 / 35.8$ & $9.1 / 9.4$ \\
\hline Mean & & $16.9 / 15.3$ & $23.5 / 14.0$ & $22.8 / 20.7$ & $28.8 / 20.5$ & $35.1 / 33.3$ & $10.1 / 8.3$ \\
\hline S.D. & & $2.41 / 1.20$ & $19.8 / 9.14$ & $2.88 / 1.73$ & $18.1 / 8.48$ & $3.75 / 2.76$ & $5.41 / 3.16$ \\
\hline \multicolumn{8}{|c|}{ Tibial nerve stimulation } \\
\hline 1 & 18.1 & $19.4 /-$ & $3.4 /-$ & $27.6 /-$ & $4.4 /-$ & $43.2 /$ & $2.8>-$ \\
\hline 2 & 20.4 & $20.5 / 19.6$ & $4.6 / 2.2$ & $25.5 / 26.8$ & $3.6 / 3.9$ & $33.3 / 41.4$ & $2.8 / 2.6$ \\
\hline 3 & 24.0 & $23.4 / 24.0$ & $8.9 / 3.0$ & $28.0 / 32.2$ & $34.8 / 6.2$ & $37.4 / 44.4$ & $2.5 / 3.4$ \\
\hline 4 & 15.4 & $18.8 / 19.0$ & $9.0 / 14.1$ & $25.4 / 25.4$ & $9.7 / 16.6$ & $40.2 / 39.8$ & $4.0 / 5.8$ \\
\hline 5 & 20.6 & $22.0 / 19.0$ & $4.7 / 1.3$ & $28.8 / 28.8$ & $6.6 / 3.1$ & $44.2 / 43.8$ & $2.9 / 2.6$ \\
\hline Mean & & $20.8 / 20.4$ & $6.1 / 5.2$ & $27.1 / 28.3$ & $11.8 / 7.5$ & $39.7 / 42.4$ & $3.0 / 3.6$ \\
\hline S.D. & & $1.89 / 2.42$ & $2.63 / 6.01$ & $1.53 / 2.95$ & $13.1 / 6.24$ & $4.44 / 2.14$ & $0.58 / 1.51$ \\
\hline
\end{tabular}

\section{LEFT}
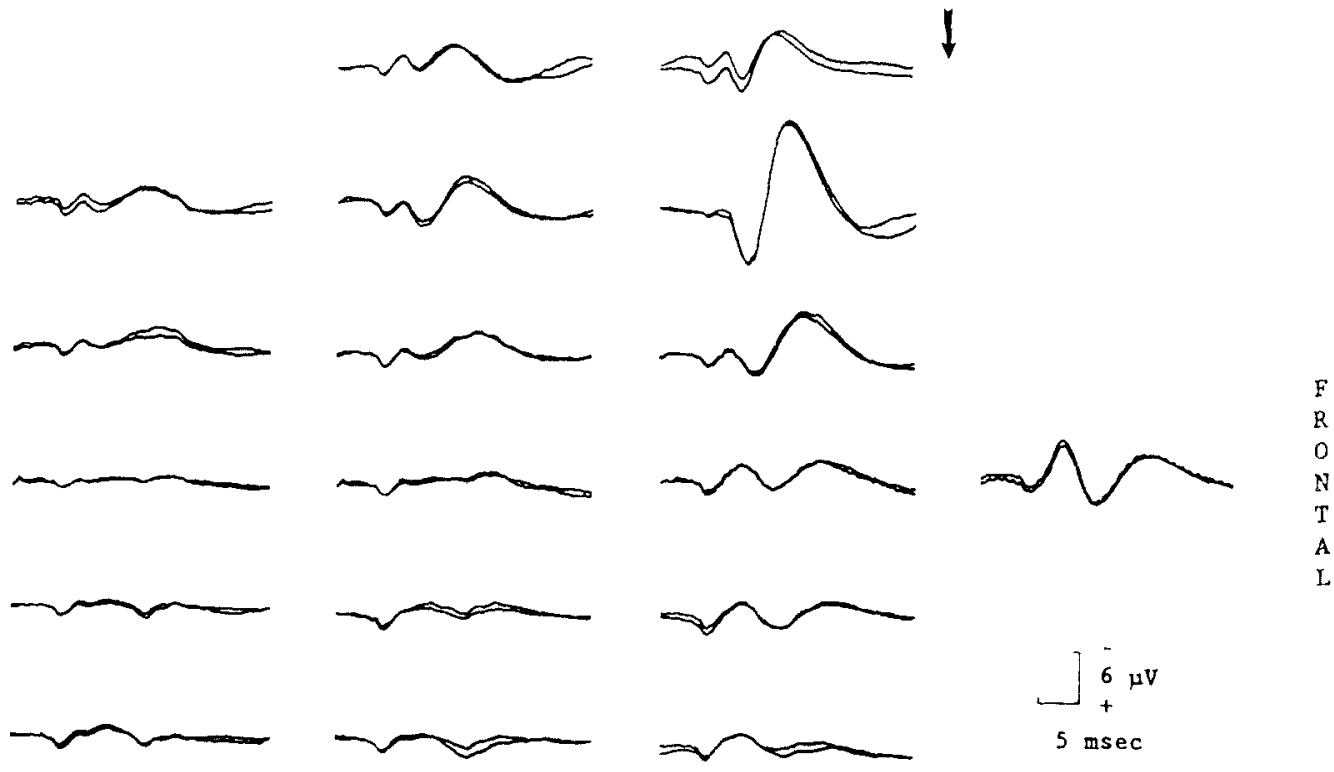

A
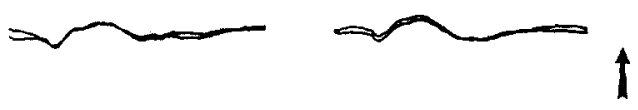

\section{RIGHT}

Fig. 3. Right median (A) and right tibial (B) SSEPs of dog no. 4, showing localization of the P1-N1-P2 complex along the coronal 
of its anterior border along the coronal (frontoparietal) suture. Tibial nerve SSEPs had more variable topography. They were always maximal near the bregma (point of crossing of the coronal and sagittal sutures), but not always located contralateral to the stimulated side. Of the 9 hindlimbs tested, tibial SSEPs were contralateral in 5 . ipsilateral in 2, and maximal at the midline in the other 2 . In both cases of an ipsilateral response, no SSEP was recorded over the contralateral hemisphere. Moreover. the topographies of left and right tibial SSEPs were of ten not mirror images of each other. In 3 of 4 dogs in which both sides were tested, left and right tibial SSEPs differed with respect to whether they were maximal at the midline or ipsilateral or contralateral to the stimulated side. But despite variable laterality, the tibial SSEP in 8 of 9 cases was at least half its maximal voltage at a midline electrode near the bregma. P1 had a more restricted distribution than N1 with either median or tibial nerve stimulation. Voltages of the P1-N1-P2 complex were usually maximal at only a single electrode and could diminish markedly less than $1 \mathrm{~cm}$ away (Fig. 4).

On median nerve stimulation. $\mathrm{P} 1$ coincided with a widespread negative potential located more anteriorly and over the midline and opposite frontal-parietral regions (Fig. 3A). This negativity was followed by a similarly distributed positivity simultaneous with $\mathrm{N} 1$. P1 and N1 voltages usually exceeded those of their opposite polarity counterparts. When changes in stimulus parameters altered $\mathrm{P} 1-\mathrm{N} 1$ voltages, parallel changes were seen in the components of opposite polarity.

At Pl onset and superimposed on its initial phase, 3 or 4 diminutive potentials were often seen (Fig. 5). These waveforms were only $1-2 \mu \mathrm{V}$, but when artifact was minimal, could be recorded over
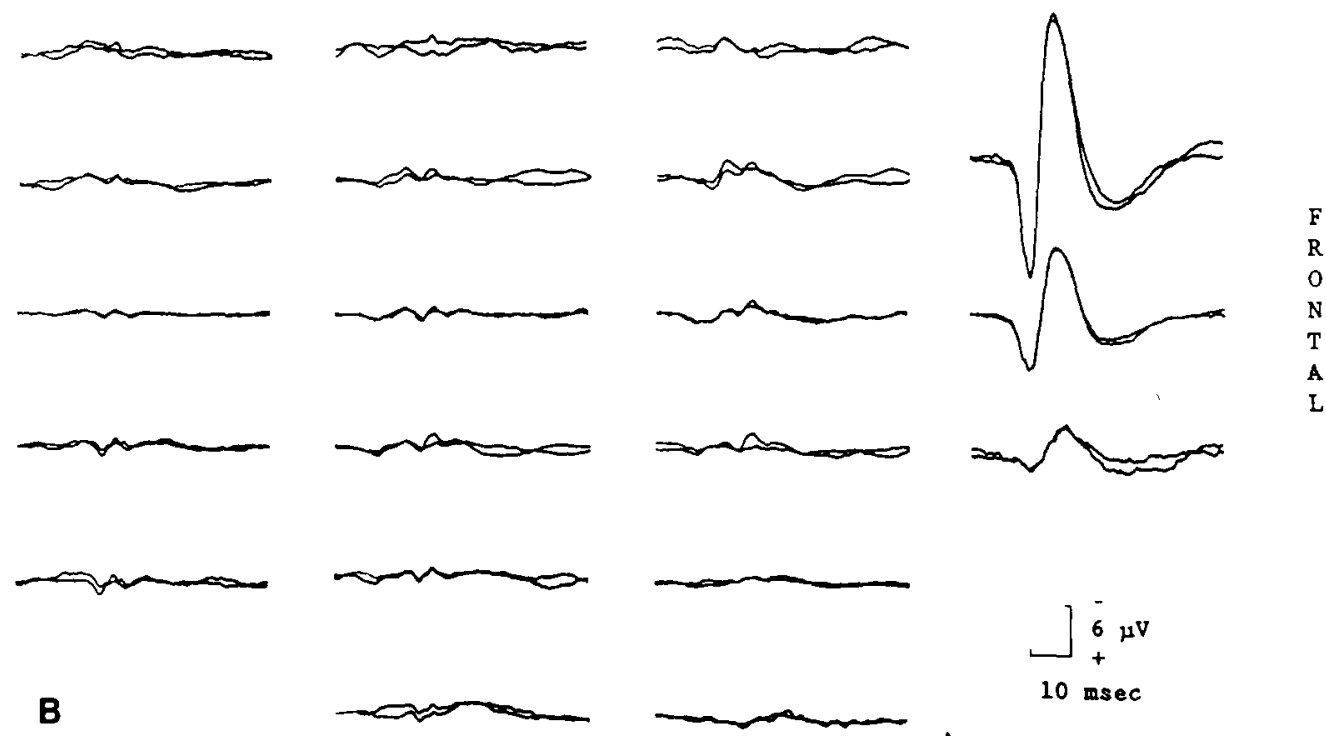

L
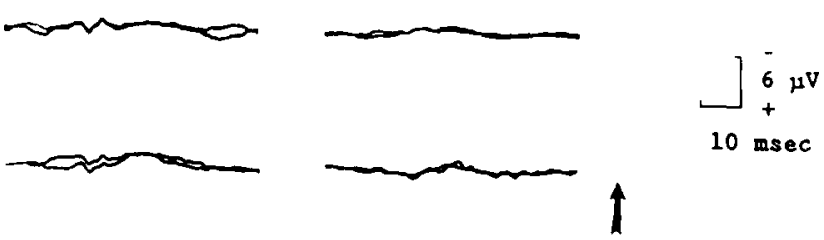

$10 \mathrm{msec}$

RIGHT

suture contralateral to side of stimulation. SSEPs are displayed as if skull faced to right, mid-dorsal recordings in middle row, and arrows denoting the plane of the coronal suture. Only the first $35 \mathrm{~ms}$ (median) or $70 \mathrm{~ms}$ (tibial) after stimulation are displayed. 


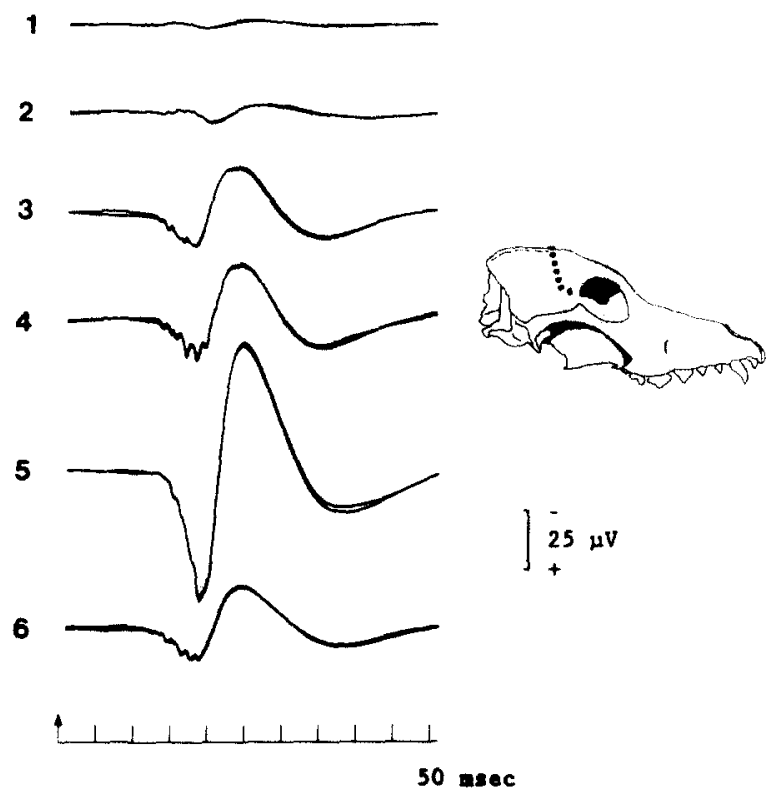

Fig. 4. Left median nerve SSEPs recorded from the anterior right parietal bone of dog no. 3, illustrating a steep voltage gradient of cortical responses. Six electrodes extended laterally from the mid-dorsal line at $8 \mathrm{~mm}$ intervals, each referenced to the inion. Cortical SSEPs were highest in voltage at electrode 5 . and at adjacent electrodes were well-defined but less than half the maximal amplitude.

A

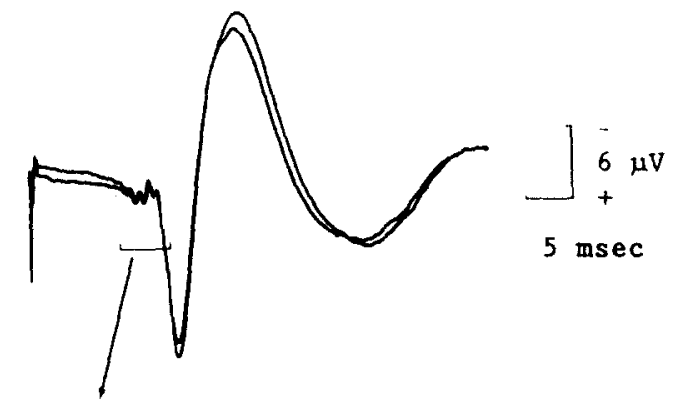

B

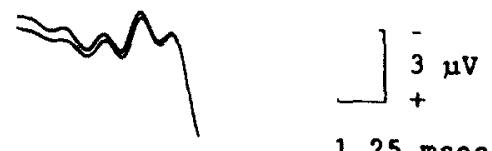

Fig. 5. Subcortical potentials preceding the cortical SSEPs on right median nerve stimulation ( $\operatorname{dog}$ no. 5 ), recorded at a standard sensitivity (A) and magnified (B). A series of 3-4 waveforms of $1-2 \mu \mathrm{V}$ with a large spatial distribution precede and coincide with the onset of $\mathrm{P} 1$.
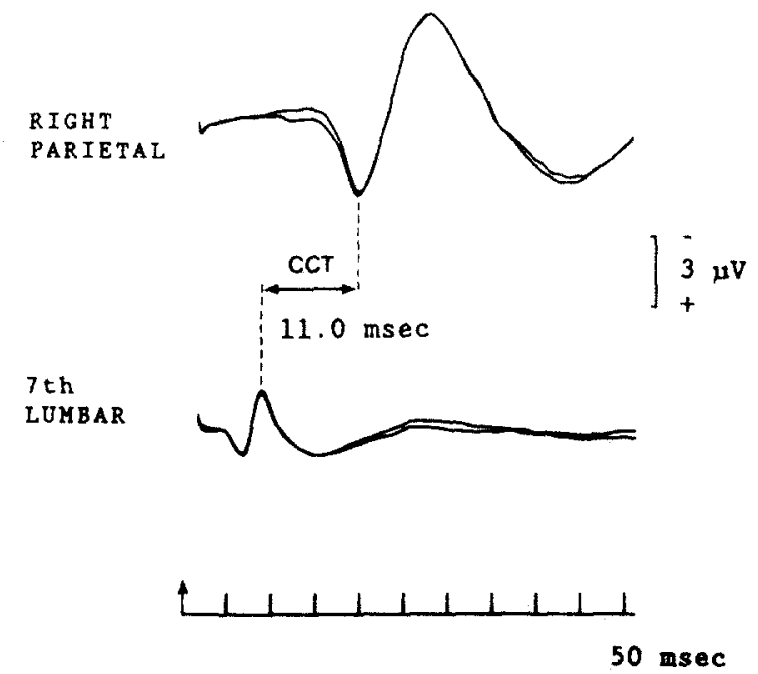

Fig. 6. Central conduction time (CCT) as determined by the difference between the peak latencies of the L7 spine potential and P1 on left tibial nerve stimulation (dog no. 1). Lower trace is from a screw electrode in the L7 spinous process referenced to the low thoracic spine. Upper trace is the cortical SSEP recorded near the bregma with an inion reference.

the entire dorsal skull, a spatial distribution broader than any other component.

Spine potentials were readily defined at the lumbar level (Fig. 6), but were usually obscured at the thoracic spine by artifacts related to respiration. No cervical spine potentials could be recorded with either subdermal needle or screw electrodes.

The effects of filter settings on median and tibial SSEPs were similar (Fig. 7). With lowfrequency filter settings of 1,5 , or $10 \mathrm{~Hz}$, SSEPs had maximal voltages and virtually identical morphologies and latencies. SSEPs were slightly attenuated with a $30 \mathrm{~Hz}$ low-frequency filter and virtually disappeared with settings of $100 \mathrm{~Hz}$ or higher, especially the P1 component. Highfrequency filters from 100 to $10,000 \mathrm{~Hz}$ yielded similar SSEP voltages, whereas a $30 \mathrm{~Hz}$ setting markedly attenuated and distorted the waveforms. The significant SSEP change at the higher settings was phase shift (Spehlmann, 1985b), with progressive increase in peak latencies as the highfrequency filter was reduced from 10,000 to 100 $\mathrm{Hz}$. The most pronounced phase shift occurred between 250 and $100 \mathrm{~Hz}$ (Fig. 7B). 


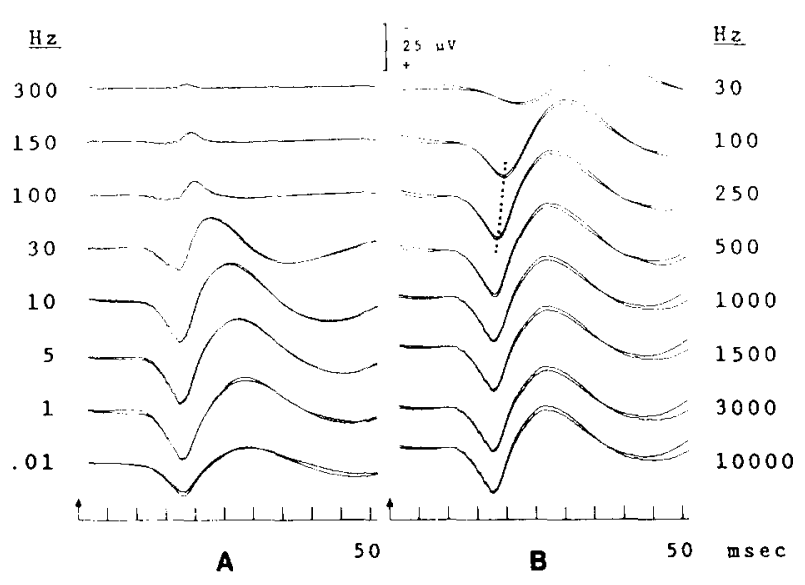

Fig. 7. Filter effects on cortical SSEPs from right median nerve stimulation of dog no. 5. A: effects of different low-frequency filter settings. High-frequency filter held constant at $3000 \mathrm{~Hz}$. B: effects of different high-frequency filter settings. Lowfrequency filter held constant at $5 \mathrm{~Hz}$. Dotted line (B) depicts a phase shift typical of analog filters. which here is greatest at high filters below $250 \mathrm{~Hz}$. A less pronounced latency increase occurs with reduction of the low filter setting.

Stimulus intensity profoundly affected median and tibial SSEP voltages (Fig. 8). At motor threshold, voltages were no more than $30-60 \%$ of their maximum values. The optimal stimulus intensity was twice median motor threshold or 3 times the tibial threshold value. The subcortical components at P1 onset were evident only with

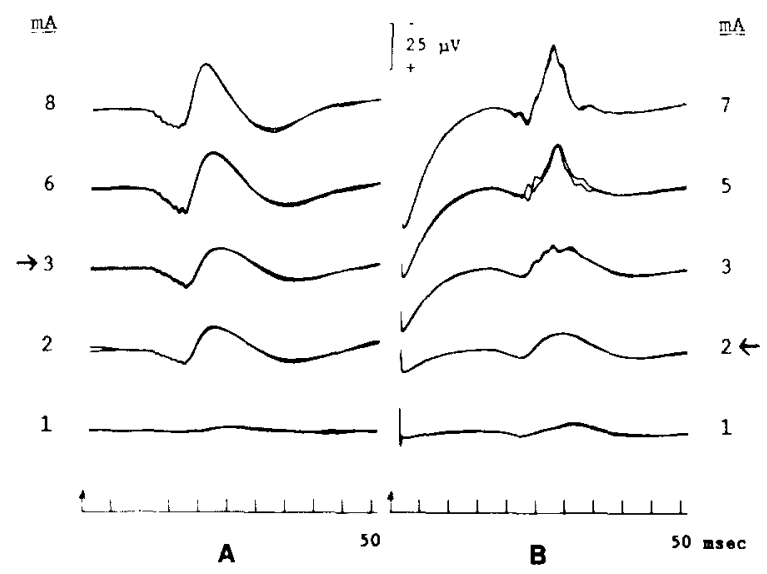

Fig. 8. Effects of stimulus intensity on cortical SSEPs from unilateral median (A) and tibial (B) nerve stimulation (dog no. 5). Arrows indicate motor threshold in $\mathrm{mA}$. Optimal SSEP voltage and definition required an intensity 2-3 times the motor threshold value.
STIMULUS

RATE $(\mathrm{Hz})$

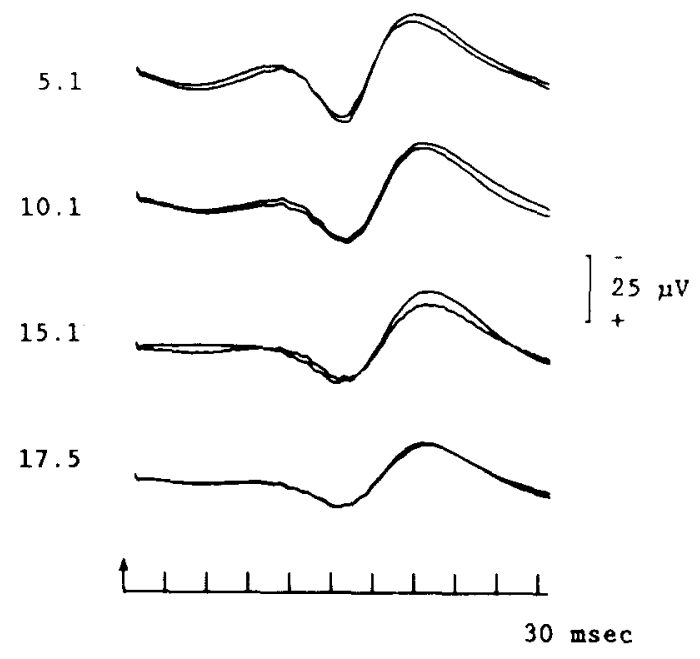

Fig. 9. Effect of stimulus rate on cortical SSEPs from right median nerve stimulation ( $\operatorname{dog}$ no. 5). As stimulus rate was increased from 5.1 to $17.5 \mathrm{~Hz}$. there was a progressive decline in $\mathrm{Pl}$ and $\mathrm{N} 1$ voltages without latency changes.

suprathreshold intensities. When the stimulating electrode was close to the nerve, the motor threshold was 1-3 mA. Intensities 2-3 times this value produced brisk, localized flexor twitches of the paw with little electrical or movement artifact.

Increasing the stimulus rate reduced the voltages of median and tibial SSEPs without affecting latencies (Fig. 9). The voltage decrement between 5.1 and $10.1 \mathrm{~Hz}$ was small, but increased with 15.1 and $17.5 \mathrm{~Hz}$ rates.

\section{Discussion}

Our study demonstrates that well-defined SSEPs can be consistently evoked from the median or tibial nerve of the dog. Voltages are enhanced if stimulation and recording parameters are properly selected. For cortical components, variations in morphology, topography, and latency are small as compared to voltage differences, which may be considerable even between left and right limbs of the same animal.

The early cortical SSEPs recorded from our 5 dogs are similar to those obtained by other investi- 
gators from dog (Bennett et al., 1977; Parker, 1978; Kornegay et al., 1981; Dong et al.. 1983; Nakagawa et al., 1986; McPherson et al., 1986; Cerchiari et al., 1987) and cat (Allison et al., 1966; Gregory et al., 1979; Allison and Hume, 1981: Dong et al., 1982; Sutton et al., 1982; Sato et al., 1984; Meyer et al., 1985). P1, N1, and P2 components are consistently recorded near the contralateral ectosylvian gyrus. Some studies (Allison et al., 1966; Gregory et al., 1979; Allison and Hume, 1981; Sato et al., 1984; Lesnick et al., 1984) have not described N1 or P2 components, probably due to use of a shorter analysis time or attenuation by anesthesia. Later cortical components N2 and P3 cannot be consistently recorded from anesthetized animals (Sutton et al., 1982).

There is no general agreement about waveform nomenclature for canine SSEPs. We adopted a purely descriptive nomenclature that is suitable for relatively stereotyped waveforms, promulgates no unwarranted assumptions about generator sources of canine SSEPs or their counterparts in man, and facilitates comparisons among species.

The attributes of $\mathrm{P} 1, \mathrm{~N} 1$, and $\mathrm{P} 2$ suggest that all are generated at a cortical level. They have circumscribed distributions over somatosensory cortex, relatively long latencies and durations, and high amplitudes. These features are consistent with the conclusion from cortical ablation studies that the anterior portion of the ectosylvian gyrus is the principal generator of the cortical SSEP (Marshall et al., 1941; Coyer et al., 1986). Voltage gradients of cortical SSEPs are steep, necessitating precise electrode positioning to record responses at their maximal amplitude. We found the coronal suture to be the most useful skull landmark in all 5 dogs.

The 3 or 4 tiny waveforms at P1 onset were low in voltage, widespread over both sides of the head with unilateral stimulation, and constant in configuration and polarity. Such attributes are consistent with subcortical potentials recorded by volume conduction at a distance from their generator sources (Spehlmann, 1985a). Iragui-Madoz and Wiederholt (1977a and b) recorded identical potentials from the skulls of cats, and demonstrated with ablation/transection studies (1977a) and with simultaneous skull and depth recordings (1977b) that they arise in cervical spinal cord, brainstem, thalamus, and subcortical white matter The application of subcortical potentials in experimental models is limited by their very low voltages. Delineating them may require bilateral stimulation, prolonged averaging, and very low levels of artifact.

Canine SSEPs have many similarities to those recorded from humans. In both species, cortical SSEPs are localized over dorsolateral somatosensory cortex on median nerve stimulation and close to the midline in tibial nerve studies. This SSEP distribution reflects the functional organization of somatosensory cortex in both species as an "inverted homunculus". Another similarity is the more variable topography of tibial nerve SSEPs. In humans these responses are often maximal ipsilateral to the stimulus (paradoxical lateralization) due to an oblique orientation of the SSEP generator to the plane of the recording electrode (Cruse et al., 1982).

One major difference between human and canine SSEPs is the polarity of the first cortical component evoked by median nerve stimulation. Whereas this potential is invariably positive (P1) in the dog, in humans it is a negativity designated N19 (Chiappa, 1983a) or N20 (American EEG Society, 1984). Although some evidence has suggested that the human $\mathrm{N} 20$ originates in the thalamus (Chiappa, 1983b), the viewpoint of many investigators favors a generator in somatosensory cortex (Emerson and Pedley, 1984). The canine P1 and human N20 appear to be equivalent as electrical manifestations of primary cortical activation. Their opposite polarities probably result from differing orientations of the SSEP generator to the cerebral convexity, reflecting the different geometry of the somatosensory cortex hand area in primates and non-primates (Allison and Hume, 1981).

Our findings and those of previous studies suggest that technical guidelines can be formulated for SSEP studies of the dog and similar species.

\section{Stimulation}

EEG needle electrodes can be inserted close to the peripheral nerve and the hub anchored to the skin with tape or suture. The median and tibial nerves are easily stimulated in the distal extremity 
because of their large size, superficial course, and proximity to readily identifiable external landmarks. They are larger than the ulnar and peroneal nerves, respectively (Miller et al., 1964b). Other peripheral nerves have been used (Parker, 1978; Gregory et al.. 1979; Kornegay et al., 1981; Dong et al., 1983; Sato et al., 1984; Nakagawa et al., 1986), but without apparent advantage for evaluating cerebral function. A stimulus duration of $200 \mu \mathrm{s}$ is suitable; with longer durations, artifact may become large. A stimulus intensity $2-3$ times the motor threshold elicits SSEPs of maximal voltage and definition, usually with minimal electrical or muscle artifact. Less than $10 \mathrm{~mA}$ were needed in our study if stimulating electrodes were positioned properly. Parker (1978) likewise reported a stimulus intensity of 3 times threshold value to be supramaximal for canine SSEPs. A stimulus rate of about $5 \mathrm{~Hz}$ elicits larger responses than $10 \mathrm{~Hz}$ or higher rates. The stimulus should produce a readily visible, brisk, well-localized twitch with flexion of the paw. When the stimulator is not close to the nerve, the threshold is higher and the twitch more diffuse, increasing stimulus and muscle artifact. The ground can be a band, plate, or needle electrode on the stimulated limb or a screw electrode in the skull.

\section{Recording}

A critical step in reducing artifact is selecting filter settings that are no wider than necessary to define the signal of interest (Lueders et al., 1985). Cortical SSEPs of the dog consist primarily of $10-250 \mathrm{~Hz}$ activity; frequencies less than $100 \mathrm{~Hz}$ provide the largest contribution. A $30 \mathrm{~Hz}$ lowfrequency filter causes some reduction of SSEP amplitude, but can be indispensable in eliminating slow wave artifacts. It proved a practical compromise between attenuation of the SSEP by filtering and its distortion by low-frequency artifacts. The high-frequency filter need be set no higher than $250 \mathrm{~Hz}$. An analysis time (sweep) of $40-50 \mathrm{~ms}$ for median nerve or $80-100 \mathrm{~ms}$ for tibial nerve studies is adequate unless SSEPs are markedly delayed. Averages of $100-300$ individual responses yield well-defined cortical waveforms from the skull when artifact levels are low.
Recording electrodes are very stable if attached to the skull. One potential difficulty is that skulls differ more in size and shape among domestic dogs than in any other mammalian species (Miller et al., 1964a). However, the coronal suture is a useful landmark for placing electrodes close to the ectosylvian gyrus. The point at which the SSEP is maximal varies somewhat, but generally lies within $2 \mathrm{~cm}$ of the bregma for tibial nerve SSEPs or $0.5-0.75$ of the distance between the sagittal and squamous sutures for median nerve SSEPs. The maximal response is best localized by exploring along the coronal suture with several electrodes. Scalp recordings from the dog - with either surface or needle electrodes - are technically difficult because of the massive temporalis muscle that invests the dorsolateral skull. Legible scalp recordings may require deep sedation of the animal or neuromuscular blockade, and lack the amplitude of SSEPs recorded from the skull.

Lumbar spine potentials evoked from the tibial nerve are readily recorded from screw electrodes in spinous processes. We were unable to identify cervical spine potentials with either median or tibial nerve stimulation. Non-invasive recording of cervical spine and medullary SSEP components is technically difficult because of the dog's massive nuchal musculature and bone structure, as well as interference from respirations and electrocardiogram. Allison and Hume (1981) succeeded with a more invasive technique in which coaxial electromyographic electrodes were inserted in or near the cord dorsum.

The posterior cranium (inion) is a stable and accessible site for the reference electrode. Nasal or frontal references appear to be equally satisfactory (Iragui-Madoz and Wiederholt, 1977a and b: Parker, 1978; Kornegay et al., 1981; Allison and Hume, 1981; Sutton et al., 1982; Dong et al., 1983; Lesnick et al., 1984; McPherson et al., 1986; Cerchiari et al., 1987). Non-cephalic references are less desirable because muscle, movement, and electrocardiogram artifacts are greater, and large inter-electrode distances often give rise to greater electrical interference (Webster, 1984).

The sensitivity and specificity of the SSEP in detecting conduction abnormality are enhanced by measuring the central conduction time (CCT). 
which approximates the transit time of the afferent volley through the spinal cord and brain. Unlike peak latencies, the CCT is minimally affected by limb length, limb temperature, or peripheral nerve lesions. The CCT of the dog is the difference between the peak latencies of the P1 component and a lumbar spine potential (Fig. 6).

Our study will hopefully encourage greater standardization of SSEP studies in animals. Selection of appropriate stimulation and recording techniques will enhance the quality and consistency of SSEPs recorded from the sedated dog, and are probably applicable to other mammalian species. Electrodes can be implanted in the sedated animal without serious morbidity. Cortical SSEPs have highly consistent morphology, topography, and latency. Because SSEP voltages can show large inter-side and inter-subject variations, measurements under experimental conditions must be compared to baseline values specific to each limb tested. Technical parameters such as bandpass, stimulus intensity and rate, and recording site affect the voltages and latencies of SSEPs. A consistent and meticulous approach will assure that SSEP changes related to physiological events can be distinguished from those caused by variations in technique.

\section{Acknowledgements}

We thank the Nicolet Biomedical for providing an electro-diagnostic system for evoked potential recordings. We are also grateful to Paul Domer, R.EEG/EP.T., for assistance in preparing the illustrations, and to Ivo Drury, M.B.B.Ch., for reviewing the manuscript.

\section{References}

Allison, T., Goff, W.R. and Sterman, M.B. (1966) Cerebral somatosensory responses evoked during sleep in the cat, Electroencephalogr. Clin. Neurophysiol., 21: 461-468.

Allison, T. and Hume, A.L. (1981) A comparitive analysis of short-latency somatosensory evoked potentials in man, monkey, cat, and rat, Exp. Neurol., 72: 592-611.

American Electroencephalographic Society (1984) Guidelines for clinical evoked potential studies, J. Clin. Neurophysiol., 1: 3-53.
Bennett, M.H., Albin, M.S., Bunegin, L., Dujovny, M., Hellstrom, H. and Jannetta, P.J. (1977). Evoked potential changes during brain retraction in dogs, Stroke, 8: 487-492

Cerchiari, E.L., Hoel, T.M., Safar, P. and Sclabassi, R.J. (1987) Protective effects of combined superoxide dimutase and deferoxamine on recovery of cerebral blood flow and function after cardiac arrest in dogs, Stroke, 18: 869-878.

Chiappa, K.H. (1983a) Evoked Potentials in Clinical Medicine. Raven, New York, p. 211.

Chiappa, K.H. (1983b) Evoked Potentials in Clinical Medicine, Raven, New York, pp. 259-262.

Coyer, P.E., Lesnick, J.E., Michele, J.J. and Simeone. F.A. (1986) Failure of the somatosensory evoked potential following middle cerebral artery occlusion and high-grade ischemia in the cat - effects of hemodilution, Stroke, 17: $37-43$.

Cruse, R., Klem, G., Lesser, R.P. and Lueders, H. (1982) Paradoxical lateralization of cortical potentials evoked by stimulation of posterior tibial nerve, Arch. Neurol., 39: 222-225.

Dong, W.K., Bledsoe, S.W., Eng, D.Y., Heavner, J.E., Shaw, C.M., Hornbein, T.F. and Anderson, J.L. (1983) Profound arterial hypotension in dogs: brain electrical activity and organ integrity, Anesthesiology, 58: 61-7.1

Dong, W.K., Harkins, S.W. and Ashleman, B.T. (1982) Origins of cat somatosensory far-field and early near-field evoked potentials. Electroencephalogr. Clin. Neurophysiol., 53: 143-165.

Emerson, R.G. and Pedley, T.A. (1984) Generator sources of median somatosensory evoked potentials, J. Clin. Neurophysiol., 1: 203-218.

Gregory, P.C., McGeorge, A.P., Fitch, W., Graham, D.I., MacKenzie, E.T. and Harper. A.M. (1979) Effects of hemorrhagic hypotension on the cerebral circulation. II. Electrocortical function, Stroke, 10: 719-723.

Iragui-Madoz, V.J, and Wiederholt, W.C. (1977a) Far field somatosensory evoked potentials in the cat. Electroencephalogr. Clin. Neurophysiol., 43: 646-657.

Iragui-Madoz, V.J. and Wiederholt, W.C. (1977b) Far-field somatosensory evoked potentials in the cat: correlation with depth recording, Ann. Neurol., 1: 569-574.

Kornegay, J.N., Marshall, A.E., Purinton, P.T. and Oliver, J.E. (1981) Somatosensory-evoked potential in clinically normal dogs, Am. J. Vet. Res., 42: 70-73.

Lesnick, J.E., Michele, J.J., Simone, F.A., DeFeo, S. and Welsh, F.A. (1984) Alteration of somatosensory evoked potentials in response to global ischemia, J. Neurosurg., 60:490-494.

Lueders, H., Lesser, R.P., Dinner, D.S. and Morris, H.H. (1985) Optimizing stimulating and recording parameters in somatosensory evoked potential studies, J. Clin. Neurophysiol., 2: 383-396.

Marshall, W.H., Woolsey, C.N. and Bard, P. (1941) Observations on cortical somatic mechanisms of cat and monkey, $J$. Neurophysiol., 4: 1-24.

McPherson, R.W., Zeger, S. and Traystman, R.J. (1986) Relationship of somatosensory evoked potentials and cerebral oxygen consumption during hypoxic hypoxia in dogs, Stroke, 17: 30-36. 
Meyer, K.L., Dempsey, R.J., Roy, M.W. and Donaldson, D.L. (1985) Somatosensory evoked potentials as a measure of experimental cerebral ischemia, J. Neurosurg., 62: 269-275.

Miller, M.E., Christensen, G.C. and Evans, H.E. (1964a) The skeletal system. In: M.E. Miller, G.C. Christensen and H.E. Evans, (Eds.) Anatomy of the Dog, Saunders, Philadelphia. p. 8 .

Miller, M.E., Christensen. G.C. and Evans, H.E. (1964b) The spinal nerves. In M.E. Miller. G.C. Christensen and H.E. Evans (Eds.) Anatomy of the Dog, Saunders, Philadelphia. pp. 586.619 .

Nakagawa, Y., Kinomoto, H. and Abe, H. (1986) Effects of dopamine on cortical blood flow and somatosensory evoked potentials in the acute stages of cerebral ischemia, Stroke. 17: $25-30$.

Parker, A.J. (1978) Evoked cisterna cerebellomedullaris potentials in the clinically normal dog. Am. J. Vet. Res., 39: $1811-1815$
Ropper, A.H. (1986) Evoked potentials in cerebral ischemia. Stroke, 17: 3-5.

Sato, M.. Pawlik. G.. Umbach. C. and Heiss, W.D. (1984) Comparative studies of regional CNS blood flow and evoked potentials in the cat: effects of hypotensive ischemia on somatosensory evoked potentials in cerebral cortex and spinal cord, Stroke, 15: 97-101.

Spehlmann, R. (1985a) Evoked Potential Primer. Butterworth. Boston, p. 11.

Spehlmann. R. (1985b) Evoked Potential Primer. Butterworth. Boston. p. 31

Sutton. L.N., Frewen, T., Marsh, R., Jaggi. J. and Bruce. D.A. (1982) The effects of deep barbiturate coma on multimodality evoked potentials, J. Neurosurg.. 57: 178-185.

Webster, J.G. (1984) Reducing motion artifacts and interference in biopotential recording. I.E..E.E.. Trans. Biomed. Eng., 31: 823-826. 